\title{
Erratum: Whole-genome mutational landscape and characterization of noncoding and structural mutations in liver cancer
}

\begin{abstract}
Akihiro Fujimoto, Mayuko Furuta, Yasushi Totoki, Tatsuhiko Tsunoda, Mamoru Kato, Yuichi Shiraishi, Hiroko Tanaka, Hiroaki Taniguchi, Yoshiiku Kawakami, Masaki Ueno, Kunihito Gotoh, Shun-ichi Ariizumi, Christopher P Wardell, Shinya Hayami, Toru Nakamura, Hiroshi Aikata, Koji Arihiro, Keith A Boroevich, Tetsuo Abe, Kaoru Nakano, Kazuhiro Maejima, Aya Sasaki-Oku, Ayako Ohsawa, Tetsuo Shibuya, Hiromi Nakamura, Natsuko Hama, Fumie Hosoda, Yasuhito Arai, Shoko Ohashi, Tomoko Urushidate, Genta Nagae, Shogo Yamamoto, Hiroki Ueda, Kenji Tatsuno, Hidenori Ojima, Nobuyoshi Hiraoka, Takuji Okusaka, Michiaki Kubo, Shigeru Marubashi, Terumasa Yamada, Satoshi Hirano, Masakazu Yamamoto, Hideki Ohdan, Kazuaki Shimada, Osamu Ishikawa, Hiroki Yamaue, Kazuki Chayama, Satoru Miyano, Hiroyuki Aburatani, Tatsuhiro Shibata \& Hidewaki Nakagawa
\end{abstract}

Nat. Genet.; doi:10.1038/ng.3547; corrected online 18 April 2016

In the version of this article initially published online, the mutation category plots in the top panel of Figure 1 were not correctly aligned with the corresponding genes. In addition, the labels for tumor types on the left were not correctly aligned with individual samples. The errors have been corrected for the print, PDF and HTML versions of this article.

\section{Corrigendum: Recurrent mTORC1-activating RRAGC mutations in follicular Iymphoma}

Jessica Okosun, Rachel L Wolfson, Jun Wang, Shamzah Araf, Lucy Wilkins, Brian M Castellano, Leire Escudero-Ibarz, Ahad Fahad Al Seraihi, Julia Richter, Stephan H Bernhart, Alejo Efeyan, Sameena Iqbal, Janet Matthews, Andrew Clear, José Afonso Guerra-Assunção, Csaba Bödör, Hilmar Quentmeier, Christopher Mansbridge, Peter Johnson, Andrew Davies, Jonathan C Strefford, Graham Packham, Sharon Barrans, Andrew Jack, Ming-Qing Du, Maria Calaminici, T Andrew Lister, Rebecca Auer, Silvia Montoto, John G Gribben, Reiner Siebert, Claude Chelala, Roberto Zoncu, David M Sabatini \& Jude Fitzgibbon Nat. Genet.; doi:10.1038/ng.3473; corrected online 12 January 2016

In the version of this article initially published online, several funding sources were omitted from the Acknowledgments section. The error has been corrected for the print, PDF and HTML versions of this article.

\section{Corrigendum: The spotted gar genome illuminates vertebrate evolution and facilitates human-teleost comparisons}

Ingo Braasch, Andrew R Gehrke, Jeramiah J Smith, Kazuhiko Kawasaki, Tereza Manousaki, Jeremy Pasquier, Angel Amores, Thomas Desvignes, Peter Batzel, Julian Catchen, Aaron M Berlin, Michael S Campbell, Daniel Barrell, Kyle J Martin, John F Mulley, Vydianathan Ravi, Alison P Lee, Tetsuya Nakamura, Domitille Chalopin, Shaohua Fan, Dustin Wcisel, Cristian Cañestro, Jason Sydes, Felix E G Beaudry, Yi Sun, Jana Hertel, Michael J Beam, Mario Fasold, Mikio Ishiyama, Jeremy Johnson, Steffi Kehr, Marcia Lara, John H Letaw, Gary W Litman, Ronda T Litman, Masato Mikami, Tatsuya Ota, Nil Ratan Saha, Louise Williams, Peter F Stadler, Han Wang, John S Taylor, Quenton Fontenot, Allyse Ferrara, Stephen M J Searle, Bronwen Aken, Mark Yandell, Igor Schneider, Jeffrey A Yoder, Jean-Nicolas Volff, Axel Meyer, Chris T Amemiya, Byrappa Venkatesh, Peter W H Holland, Yann Guiguen, Julien Bobe, Neil H Shubin, Federica Di Palma, Jessica Alföldi, Kerstin Lindblad-Toh \& John H Postlethwait Nat. Genet. 48, 427-437 (2016); published online 7 March 2016; corrected after print 25 April 2016

As we intended, other researchers have been able to use the draft spotted gar genome sequence available from the Broad Institute website since December 2011, the assembly LepOcu1 publicly available from NCBI since 13 January 2012 under accession code GCA000242695.1, and the Ensembl gene annotation (version 74, December 2013; http://www.ensembl.org/Lepisosteus_oculatus/Info/Annotation) and recent annotation by NCBI on 15 May 2014 guided by RNA sequence data from ten tissues. While this article was in review, a paper (Nature 526, 108-111, 2015) was published that arrives at conclusions similar to some of our own analyses, and we wish to acknowledge that publication, which used our unpublished data and genome annotations, emphasizing the importance of the strategy of early release of sequence data. The correction has been made to the HTML and PDF versions of the article. 